\title{
Deflating Link Buffers in a Wireless Mesh Network
}

\author{
Kamran Jamshaid ${ }^{\mathrm{a}, 1}$, Basem Shihada ${ }^{\mathrm{a}}$, Ahmad Showail ${ }^{\mathrm{a}}$, Philip Levis ${ }^{\mathrm{b}}$ \\ ${ }^{a}$ King Abdullah University of Science and Technology, Thuwal, Saudi Arabia \\ ${ }^{b}$ Stanford University, Stanford, CA, USA
}

\begin{abstract}
We analyze the problem of buffer sizing for backlogged TCP flows in 802.11based wireless mesh networks. Our objective is to maintain high network utilization while providing low queueing delays. Unlike wired networks where a single link buffer feeds a bottleneck link, the radio spectral resource in a mesh network is shared among a set of contending mesh routers. We account for this by formulating the buffer size problem as sizing a collective buffer distributed over a set of interfering nodes. In this paper we propose mechanisms for sizing and distributing this collective buffer among the mesh nodes constituting the network bottleneck. Our mechanism factors in the network topology and wireless link rates, improving on pre-set buffer allocations that cannot optimally work across the range of configurations achievable with 802.11 radios. We evaluate our mechanisms using simulations as well as experiments on a testbed. Our results show that we can reduce the RTT of a flow by $6 \times$ or more, at the cost of less than $10 \%$ drop in end-to-end flow throughput.
\end{abstract}

Keywords: Wireless Mesh Networks, Buffer Size, TCP

\section{Introduction}

Packet-switched networks use buffers to accommodate transient traffic bursts. These buffers aim to prevent packet loss and maintain high output link utilization. Buffer sizing is an important network configuration parameter: under-

\footnotetext{
Email address: kamjam@acm.org (Kamran Jamshaid)

${ }^{1}$ Phone: +966-2-808-0398, Fax: +966-2-808-0332
} 
buffered networks lead to frequent packet loss and subsequent under-utilization of network resources, while over-buffered networks lead to increased queueing delays.

We wish to study the impact of buffer sizing in 802.11-based Wireless Mesh Networks (WMNs) providing backhaul connectivity for last mile Internet access [20]. These multihop networks have stationary mesh routers affixed to rooftops and building structures, forming a multihop wireless backhaul. Client devices connect to their preferred mesh router via wire or an orthogonal wireless channel. The capacity of this link is higher than the capacity achievable along the multi-hop path of wireless mesh routers, and thus buffers are more likely to build up at the mesh routers. Most of the traffic in this network is directed towards or away from the gateway mesh router $(\mathrm{GW})$ that bridges traffic to the wired Internet.

Buffers accure at network bottlenecks where the ingress rate is typically much higher than the egress rate. Much of prior research on buffer sizing is for core Internet routers [11]. However, with the core capacities increasingly being over-provisioned, the bottleneck often lies in the last mile access network, e.g., in a typical home network, a high-speed Wi-Fi router (with $802.11 \mathrm{n}$ rates of $150 \mathrm{Mb} / \mathrm{s}$ and higher) feeds a cable or DSL modem with 1-2 Mb/s provisioned link capacity. Similarly, in WMNs, capacity bottlenecks (and subsequent queue buildups) are more likely to be the mesh routers that share the wireless spectrum with other mesh devices. While these mesh nodes carry only a fraction of the flows compared to core Internet routers, yet they have a number of unique challenges:

- In a wired network, buffer sizing is usually studied in the context of keeping the bottleneck link fully utilized. Wireless links, however, are just an abstraction for shared spectrum between communicating entities and their neighbors, only one of which can successfully transmit at a time. It is not clear how buffers can be associated with a distributed set of nodes, some of which relay traffic for other nodes in the network. 
- The capacity of the shared spectrum is not constant, but varies over time due to sporadic noise and interference from other devices. Further, the packet inter-service time of CSMA/CA radios varies due to the random scheduling by the MAC layer.

With declining memory chip prices, system architects may be inclined to over-provision routing buffers. This, however, does not necessarily lead to improved network performance, as also highlighted in the Bufferbloat project [3]. IP networks, in general, should minimize buffering and queueing delays. While this is apparent for real-time flows or short-lived TCP flows, it also holds for scenarios where throughput has precedence over delay (e.g., bulk file transfer with TCP). TCP's additive-increase multiplicative-decrease (AIMD) rate control algorithm is deliberately designed to fill any buffer and invoke an occasional packet loss so as to provide feedback to the sender [11]. Large network buffers increase the delay before this congestion information can be communicated to the TCP source. This has a number of ramifications: first, it can potentially affect the stability characteristics of the TCP stream [26]; and second, it increases the delay for other flows (both real-time and short-lived TCP) sharing the buffer with this stream. We illustrate these delays in Fig. 1 for a TCP flow between two nodes in a Linux-based WMN testbed in our university campus. The mesh routers use the default buffer sizes in the stock kernel. Most modern operating systems (including Linux) enable TCP window scaling by default for efficient data transfer in networks with high bandwidth-delay product (BDP). With large buffers, this allows TCP congestion window to grow to large values before registering a packet drop due to queue overflows. These packets are queued at mesh router link buffers, thus increasing queueing delays beyond 600 $\mathrm{ms}$ in this experiment. Such delays are catastrophic for real-time flows (e.g., VoIP) and can also impact the stability characteristics of TCP.

There is limited available work on the impact of buffer size on wireless network performance (see Sect. 2 for related work). Unsurprisingly, we found a significant disparity in the buffer size used in various research platforms. Many 

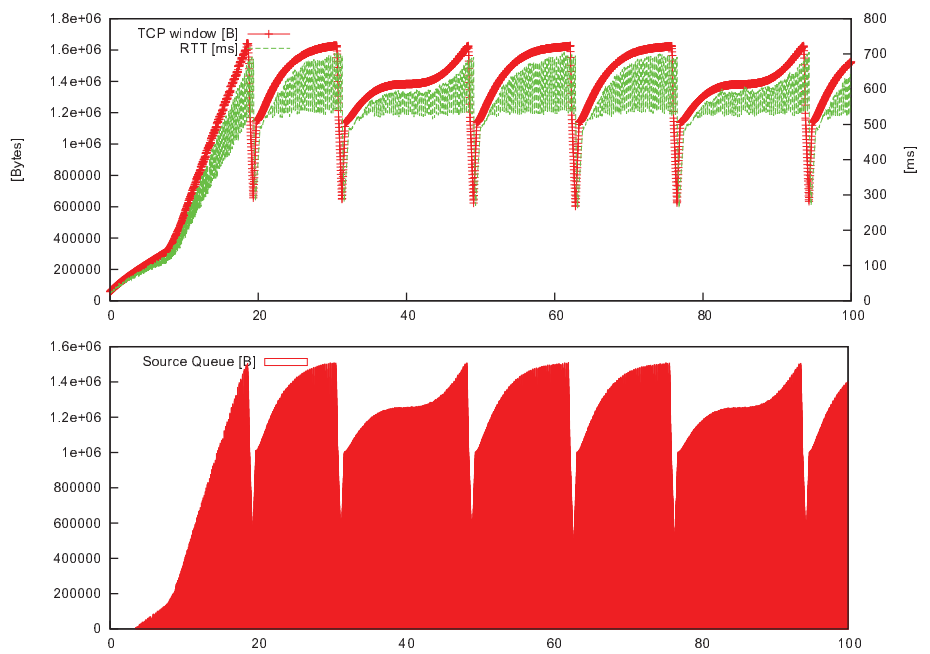

Figure 1: TCP congestion window, RTT, and egress queue utilization for a TCP flow betweeen two nodes in our testbed connecting at a link rate of $36 \mathrm{Mb} / \mathrm{s}$. The wireless nodes use default buffer sizes as in stock Linux kernel.

papers using the $n s-2[6]$ simulator use a buffer size of 50 packets, the default size for the queue object in ns-2. The legacy open source MadWifi drivers [5] for Atheros chipset use a transmission buffer of 200 packets. The new ath5k drivers [1] for the same hardware divide this 200 packet buffer equally among four queues representing the traffic access categories (ACs) as defined in Enhanced Distributed Channel Access (EDCA) mechanism. The ath9k [2] drivers for $802.11 \mathrm{n}$ Atheros radios use a buffer of 512 packets equally divided among the four ACs.

In this work we study the buffer sizing problem in WMNs using the notion of distributed queues spread over a contention neighborhood. For a TCP flow traversing multihop wireless links, the end-to-end rate is determined by the contention neighborhood that allocates the minimum transmission time to member links. For optimal network utilization, this bottleneck contention neighborhood needs to be fully utilized. We thus map the buffer sizing problem as a distributed buffer management problem for nodes sharing the bottleneck contention neighborhood. A packet transmission by any of these nodes fully utilizes the bottleneck spectral resource for the duration of this transmission. We pro- 
pose mechanisms for sizing and distributing these buffers to keep the bottleneck close to full utilization while minimizing queueing delays. Our mechanism considers the network topology and wireless link rates, and is thus adaptive to changing network conditions. In contrast, a fixed, pre-set buffer size does not suffice across the range of configurations achievable with 802.11 platform [29]; e.g., a single $802.11 \mathrm{n}$ radio interface can connect at link rates varying from 600 $\mathrm{Mb} / \mathrm{s}$ (with 4 spatial streams) to $1 \mathrm{Mb} / \mathrm{s}$ (backward compatibility with 802.11b), and thus requires widely different buffer sizes for saturating the wireless channel. Using analysis and performance evaluation with simulations and a wireless testbed, we show that our proposed scheme reduces the end-to-end delay of a TCP flow by a factor of $6 \times$ or more for the scenarios evaluated in this paper, while maintaining close to full network utilization.

Configuring a mesh router buffer introduces a number of practical considerations. Packets in a contemporary modern operating system (OS) encounter buffers at multiple layers in the software stack. Each of these buffers may introduce queueing delays. In this paper, we describe the major buffers encountered by a packet as it traverses a Linux host and discuss the performance implications of sizing these buffers. We note that while we focus on the Linux kernel, the impact of multi-layered buffering is also present in BSD and Windows-based systems. Thus some of our design guidelines are also applicable in those systems.

Our main contributions in this paper are as follows:

1. We account for the shared nature of wireless spectrum by formulating the buffer sizing problem in WMNs as sizing a distributed queue spread over multiple nodes constituting the bottleneck radio neighborhood.

2. Using a simple cost function, we propose mechanisms for sizing the transmission buffer at individual nodes in the bottleneck neighborhood queue.

3. We identify the major layers of buffers encountered by a packet through a Linux host and discuss the performance implications of their size.

4. Using simulations and an experimental testbed, we verify that our approach achieves close to full network utilization with a significant reduc- 
tion in the end-to-end delay for a flow.

The remainder of this paper is organized as follows. We first cover the background work, contrasting it with our approach. In Sect. 3 we describe the notion of a wireless network bottleneck spanning multiple, distributed nodes that limits the end-to-end rate of a multihop flow. In Sect. 4, we formulate a model for sizing the distributed buffer collectively and then allocating it among the nodes. In Sect. 5 we describe the packet transmission buffers in ns-2 mobile nodes and Linux kernel and discuss various design considerations in optimizing these buffers. We then show the efficacy of our model via simulations and experimental evaluation on a 802.11s WMN testbed. We conclude by observing what issues remain open.

\section{Related Work}

TCP's window-based congestion control algorithm can trigger a burst of packet transmissions (e.g., on receiving a cumulative ACK or several back-toback ACKs). Sustained bursts can lead to packet losses with subsequent drop in throughput. TCP pacing [35] addresses this by spacing out the transmission of a cwnd worth of packets over the estimated RTT interval, i.e., the packets are injected into the network at a rate cwnd/RTT. While this minimizes traffic burstiness (and subsequent queueing delays), it may reduce throughput compared to unmodified TCP because of delayed congestion signals and synchronized losses [10]. ElRakabawy et al.[18] observe that the inherent variability in the RTT of multihop wireless flows may offset these synchronization issues. They propose a rate-based transmission algorithm over TCP called TCP with Adaptive Pacing (TCP-AP): instead of spacing the transmission of cwnd worth of packets over the RTT of a flow, the transmission rate is computed using 4-hop propagation delay (determined by spatial reuse in a chain of wireless nodes) and coefficient of variation of recent RTTs (to identify incipient congestion along network path). We compare our proposed buffer sizing strategy against TCP-AP in Sect. 6. 
Buffer sizing has been extensively studied for wired networks. A widely used rule-of-thumb is to have buffers larger than the BDP of the network [33], i.e., $B \geq R T T \times C$, where $C$ is the bottleneck link capacity along the path, and $R T T$ is the effective round-trip propagation delay through the bottleneck link. This buffer size $B$ reflects the number of packets queued at a bottleneck link to keep it fully utilized while a TCP source recovers from a loss-induced window reduction. This rule-of-thumb holds for a single TCP flow in a wired network. When a large number of flows share a bottleneck router, the window size processes quickly synchronize in lockstep. As a result, the aggregate window size is still a sawtooth pattern, and the BDP guideline for sizing the bottleneck buffer still holds. However, when the flows are desynchronized and the window processes are independent, the buffer size $B$ can be reduced to $B=R T T \times C / \sqrt{N}$ while still achieving near 100\% utilization [11]. Enachescu et al. [19] suggest that $B$ can further be reduced to $O(\log W)$, where $W$ is the window size of each flow, resulting in buffer sizes of only $10-20$ packets while achieving $85-90 \%$ of link utilization.

Calculating the BDP of a multihop wireless network is different from a wired network because of coupling between the bandwidth and delay of a wireless link [15]. In long-haul wired links, the transmission delay is small compared to the propagation delay; thus a source can often inject multiple packets back-toback into the pipe. The same is not true for CSMA/CA-based wireless links; first, the transmitter has to contend for channel access for each transmission, and second, the 802.11 transmitter requires an ACK before it can transmit the next frame. As a result, the delay of transmitting a packet is strongly coupled with the link's effective bandwidth. Chen et al. [15] show that the BDP of multihop wireless flow is tied to the number of round-trip hops along the path to the destination. They use this BDP value to define the TCP congestion window limit in order to avoid the window overshooting problem that may self-interfere with a flow's transmission.

There is a limited amount of work on buffer sizing for wireless networks. For single-hop 802.11 WLANs, large Access Point (AP) buffers can improve fairness 
between upstream and downstream TCP flows [13]; a large buffer increases the queueing delay for TCP ACKs being transmitted back to the upstream wireless source, in effect rate limiting the upstream flows. This scheme, however, disturbs the tight feedback loop necessary for optimal operation of TCP. The AP buffer can be sized dynamically [29] to strike a balance between channel utilization and delay requirements of various flows.

For multihop wireless networks, a number of publications (e.g., [23]) propose using queue sizes for detecting network congestion and enforcing subsequent rate adjustments. $\mathrm{Xu}$ et al. [34] have extended this notion to a distributed neighborhood queue. Their NRED algorithm improves flow rate fairness by probabilistically dropping packets when the cumulative size of this distributed queue exceeds a threshold. In prior work [25], we propose sizing this neighborhood buffer to optimize the performance of TCP. This current paper extends our prior work with additional simulations as well as a testbed implementation and analysis.

Dousse [17] proposes essentially bufferless relay nodes for a MAC protocol that, among other characteristics, self-regulates the UDP traffic it injects in the network. We instead consider the 802.11 MAC protocol for WMNs and size the mesh router buffers for bursty TCP traffic.

\section{Bottleneck node buffers in a WMN}

We now first identify the bottlenecks that limit the end-to-end rate of a multihop wireless network. We then show how distributed buffers are associated with this bottleneck.

\subsection{Bottleneck collision domain}

The wireless medium is a shared resource. This limits the set of nodes that can transmit concurrently. We use the notion of collision domain [27] to identify these interfering links. The collision domain of link $l_{i}$ is defined as the set of all links that contend with $\operatorname{link} l_{i}$. In general, identifying the interfering 
links is a hard problem, as links exhibit varying degree of interference [30]. Additional mechanisms requiring active or passive monitoring may have to be used [28]. In this work we use the two-hop interference model [12] to identify interfering links; two links interfere if they operate on the same channel and one endpoint of one link is within the transmission range of one endpoint of the other link. The two-hop model approximates the interference avoidance in a 802.11 network with RTS/CTS enabled. While the model has limitations in representing complex topologies, we simply use it for ease of exposition and our proposed buffer sizing mechanism is independent of the underlying interference model. In Fig. 2, the collision domain of link $l_{3}$ includes links $l_{1}, l_{2}, l_{4}$, and $l_{5}$. We note that this notion of interference domain overestimates the impact of interference by limiting the number of nodes that can concurrently transmit. However, the actual interference between nodes is determined by the interference range, which is typically longer than the 1-hop communication range. The combined model offers acceptable accuracy with computational simplicity and practical feasibility.

The utilization of a collision domain is the sum total of transmission times for all links in a collision domain. The feasibility constraints on scheduling require that this utilization cannot exceed 1. Mathematically, we represent it as follows: Let $R_{(m, n)}$ be the link rate between neighboring nodes $(m, n)$ and let $r_{(m, n)}$ be the traffic carried by this link. Let $r_{i}$ be the end-to-end rate for flow $f_{i}$. Then $r_{(m, n)}=\sum_{i: f_{i} \text { traverses }(m, n)} r_{i}$. Let $\mathbf{C}=\left\{C_{1}, C_{2}, \ldots, C_{j}\right\}$ be the set of $j$ collision domains in this network. Ignoring physical and MAC layer headers, the feasibility constraints require

$$
\sum_{\forall(m, n) \text { in } C_{p}} \frac{r_{(m, n)}}{R_{(m, n)}} \leq 1, \forall p \in\{1,2, \ldots, j\}
$$

A saturated collision domain is defined as a collision domain which is fully utilized. A saturated collision domain becomes a bottleneck for a flow if that flow has a maximal rate among all other flows using this collision domain. A 


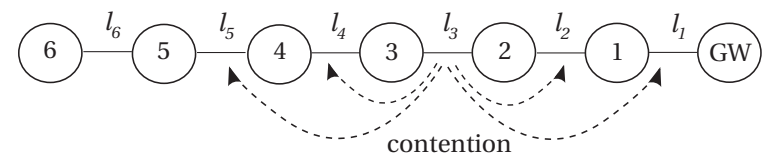

Figure 2: With a two-hop interference model, the collision domain of link $l_{3}$ includes links $l_{1}, l_{2}, l_{4}$, and $l_{5}$.

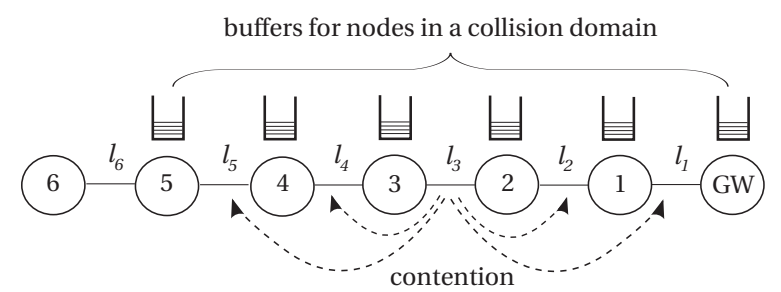

Figure 3: The distributed neighborhood buffer of a collision domain includes the buffers associated with the constituent nodes in that collision domain.

multihop flow may be part of one or more collision domains; its end-to-end rate is then bound by the collision domain that assigns it the lowest rate.

\subsection{Distributed neighborhood buffers}

Since the bottleneck collision domain limits the end-to-end rate of a multihop flow, it is important that the spectral resource in this bottleneck be fully utilized. From a buffering perspective, this is achieved if at least one node in the bottleneck always has packets to send. A packet transmission by any of these nodes fully utilizes the available spectral resource for the duration of the transmission. Thus, instead of considering buffers at individual nodes, we propose sizing the collective buffer of the bottleneck collision domain so as to saturate its available spectral resource. This neighborhood buffer is distributed over the set of nodes constituting the bottleneck. Its size is the sum of the buffer sizes of all the constituent nodes. Similarly, its packet arrival rate is the sum of the arrival rates at individual nodes. However, this neighborhood buffer does not exhibit the FIFO characteristics due to distributed queues and stochastic scheduling of 802.11 MAC protocol. In Fig. 3, we show the distributed FIFO buffer at nodes constituting the bottleneck collision domain for a 6-hop chain topology.

We note that our definition of distributed FIFO buffers includes all packets queued at a node. For Fig. 3, this includes the packets queued at node 5 
for transmission to node 6 via link $l_{6}$. This can be mitigated by maintaining a virtual per-link queue at each node, and including only the virtual queues in the distributed buffer that directly correspond to the links in the collision domain. In this current work, however, we include the entire FIFO buffer at a node in the distributed queue. This allows node 6 to transmit to 5 (but not vice versa) when there is an active transmission on link $l_{3}$. Limiting node 5 's transmission to 6 over-estimates the impact of interference when 3 transmits to 2 , but may be necessary to prevent interference at 3 when it receives a packet from 2 (as interference range is typically larger than transmission range, a concurrent transmission from node 5 may corrupt reception at node 3 ).

One simple (though naïve) way to enhance the utilization of bottleneck spectrum is to make the bottleneck neighborhood buffer arbitrarily large, and then hope that it will always have sufficient packets in queue to saturate the bottleneck even when the TCP source recovers from a packet loss. However, it is easy to visualize that if this buffer is any larger than the rate supported by the network, it will only add to the queueing delay experienced by the flow. At the same time, if the bottleneck is under-buffered, it will not always be fully utilized. Our challenge, thus, is to determine the size of this neighborhood buffer that maintains the bottleneck near $100 \%$ utilization while minimizing the queueing delay.

\section{System Model}

Consider a WMN with $\mathbf{N}=\left\{n_{1}, n_{2}, \ldots, n_{N}\right\}$ be the set of wireless mesh routers. Assume that the set $\mathbf{M}$, where $\mathbf{M} \subseteq \mathbf{N}$, represents the set of nodes in the bottleneck collision domain. Let $M=|\mathbf{M}|$, i.e., a node in a bottleneck collision domain contends with $M-1$ other nodes for channel access. The

node $n_{i}$ has a buffer of size $b_{i}$ packets. Let $B=\sum_{\forall i \text { in M }} b_{i}$ be the cumulative distributed buffer for this collision domain.

In our analysis below, we first determine $B$, and then propose a model for distributing it as $b_{i}$ units among the $M$ contending nodes. 


\subsection{Neighborhood buffer size B}

Consider a single multihop TCP stream. Assume our wireless network can support this stream at a maximum rate of $\lambda$ packets/s. ${ }^{2}$ Let the stream be in AIMD congestion avoidance phase. The TCP window size reaches a value of $W_{\max }$ packets before experiencing a loss. As a result, the sender halves the window size to $W_{\max } / 2$ packets. Since the window size limits the number of unacknowledged packets in flight, the sender, on average, waits for a time interval $\frac{W_{\max } / 2}{\lambda}$ before starting to retransmit. At the same time, the distributed buffer $B$ takes $B / \lambda$ s. to drain. For full utilization of the bottleneck spectrum, the source should start retransmitting before the buffer is fully drained, i.e., $\frac{W_{\max } / 2}{\lambda} \leq B / \lambda$, or

$$
B \geq \frac{W_{\max }}{2}
$$

When the TCP source starts retransmitting, its rate (i.e., cwnd/RTT) should be higher than $\lambda$, as otherwise the network capacity is under-utilized. Thus $\frac{W_{\max } / 2}{R T T} \geq \lambda$, or,

$$
\frac{W_{\max }}{2} \geq R T T \cdot \lambda
$$

From Eq. (1) and (2),

$$
B \geq R T T \cdot \lambda
$$

Eq. (3) is similar to that of wired networks [11]. The main difference is in $\lambda$, the maximum carrying capacity of the network. In wired networks, $\lambda$ is determined by the bottleneck link capacity. In multihop wireless networks, it is limited by the bottleneck collision domain. The exact values of $\lambda$ and $R T T$ depend on the network topology and wireless channel characteristics. Per Eq. (3), however, we size our distributed buffer $B$ higher than these instantaneously precise

\footnotetext{
${ }^{2}$ We use packets instead of bits for ease of exposition.
} 


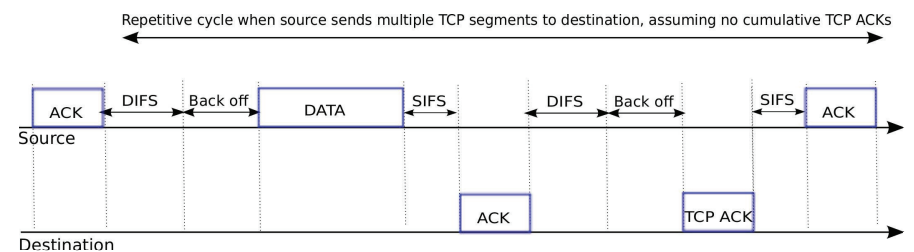

Figure 4: 802.11 MAC overhead per TCP segment transmission.

values. This is necessary to account for the time-varying capacity of the wireless channel, and non-deterministic 802.11 Distributed Coordination Function (DCF) scheduling. In this work we estimate loose upper bounds on $\lambda$ and RTT for a given network. The resulting larger-than optimal buffer trades-off queueing delay for higher channel utilization. Our results in Sect. 7 confirm that the impact of this trade-off is minimal, and we can still maintain buffers as small as 2-3 packets at most mesh nodes.

For a given flow, the upper bound on $\lambda$ is obtained when there is no competing traffic. Let the wireless link rates for the $M$ nodes in the collision domain be represented by the vector $\mathbf{W}=\left\{w_{1}, w_{2}, \ldots, w_{M}\right\}$. The bottleneck bandwidth of the path is determined by $w_{\min }=\min \left(w_{1}, w_{2}, \ldots, w_{M}\right)$. We use $\lambda=w_{\min }$ as a loose upper bound on the network carrying capacity.

The RTT in Eq. (3) should only include the propagation delay (and not the queueing delays) for the wireless links. The propagation delays are dominated by transmission delays in wireless networks. Where applicable, other delays, such as those introduced by access links bridging to the wired Internet (e.g., DSL lines), may also need to be included. To compute the wireless transmission delays, consider the MAC overhead for transmitting a TCP segment and its associated ACK in Fig. 4. Let $T_{d-D A T A}$ and $T_{d-A C K}$ represent the total transmission time for TCP segment and ACK, respectively.

$$
\begin{gathered}
T_{d-D A T A}=T_{B O}+T_{D I F S}+T_{D A T A}+T_{S I F S}+T_{A C K} \\
T_{d-A C K}=T_{B O}+T_{D I F S}+T_{T C P-A C K}+T_{S I F S}+T_{A C K}
\end{gathered}
$$

where $T_{B O}$ is the backoff interval. The average $T_{B O}, \overline{T_{B O}}=C W \times T_{\text {slot }} / 2$, where 


\begin{tabular}{|cc|}
\hline Parameter & Value \\
$T_{\text {slot }}$ & $20 \mu \mathrm{s}$ \\
$T_{S I F S}$ & $10 \mu \mathrm{s}$ \\
$T_{D I F S}$ & $50 \mu \mathrm{s}$ \\
$C W_{\min }$ & 31 \\
$C W_{\max }$ & 1023 \\
\hline
\end{tabular}

Table 1: System parameters of IEEE 802.11b [24].

$C W$ is the MAC contention window and $T_{\text {slot }}$ is the slot duration. $T_{S I F S}$ and $T_{D I F S}$ are the SIFS and DIFS deferral, respectively. $T_{D A T A}, T_{T C P-A C K}$, and $T_{A C K}$ are the transmission times to transmit a TCP segment, ACK, and MAClevel ACK, respectively. Based on the configured RTS threshold, additional time of $T_{R T S}+T_{C T S}+2 \times T_{S I F S}$ may be added to $T_{d-D A T A}$ as well as $T_{d-A C K}$, where $T_{R T S}$ and $T_{C T S}$ is the time to transmit an RTS and a CTS frame, respectively. We list the various 802.11 b system parameters in Table 1. For transmitting a TCP segment of size 1460 B and its ACK (per the exchange shown in Fig. 4), it takes approximately $15 \mathrm{~ms}$ at $1 \mathrm{Mb} / \mathrm{s}$ link rate and $2.7 \mathrm{~ms}$ at $11 \mathrm{Mb} / \mathrm{s}$ link rate.

RTT through the bottleneck collision domain can then be computed as follows:

$$
\begin{aligned}
R T T & =\sum_{i=1}^{M} T_{d-D A T A}+T_{d-A C K} \\
& =M \cdot\left(T_{d-D A T A}+T_{d-A C K}\right)
\end{aligned}
$$

\subsection{Distributing the neighborhood buffer among nodes}

Once the neighborhood buffer size $B$ is computed, we distribute it among the set of nodes in the bottleneck collision domain. One simple strategy is to divide $B$ equally among the nodes. However, this does not consider the fact that a queue drop closer to the source has consumed fewer network resources than a queue drop near the destination. In our model we introduce a cost function to capture this bias.

Fig. 5 shows the queue occupancy state transition for a mesh node. A 


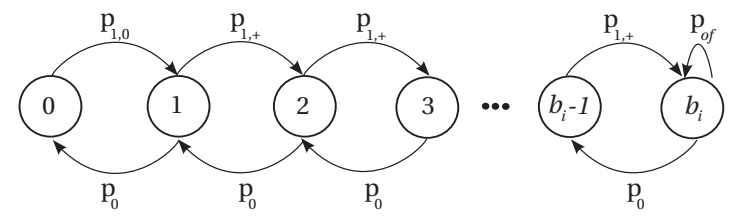

Figure 5: Queue occupancy state transition for a wireless node $n_{i}$ with a buffer of size $b_{i}$.

node $n_{i}$ can queue at most $b_{i}$ packets corresponding to its allocated buffer. Let $\pi_{k}$ represent the steady state probability that the node queue size is $k$, for $0 \leq k \leq b_{i}$. In particular, $\pi_{0}$ and $\pi_{b_{i}}$ represent the probabilities that the buffer is empty or full, respectively. The node successfully transmits a packet with probability $p_{0}$, transitioning to a state with one less packet queued. With probability $p_{1,+}$, a relay node receives a packet from its parent in the routing tree, and then queues it locally for transmission to the next hop. The transition probability $p_{1,0}$ is a special case for packet reception when the current queue size is zero. Finally, probability $p_{o f}$ represents a packet drop due to buffer overflow.

A node can transmit a packet with probability 1 if all other nodes have an empty buffer (i.e., they are in state $\pi_{0}$ ); else it contends fairly for the channel with other nodes that also have packet(s) buffered for transmission. Thus,

$$
\begin{aligned}
p_{0} & =1 \cdot \pi_{0}^{M-1}+\frac{1}{2} \cdot\left(\begin{array}{c}
M-1 \\
1
\end{array}\right) \cdot \pi_{0}^{M-2}\left(1-\pi_{0}\right) \\
& +\frac{1}{3} \cdot\left(\begin{array}{c}
M-1 \\
2
\end{array}\right) \cdot \pi_{0}^{M-3}\left(1-\pi_{0}\right)^{2}+\ldots+ \\
& +\frac{1}{M} \cdot\left(\begin{array}{c}
M-1 \\
M-1
\end{array}\right) \cdot\left(1-\pi_{0}\right)^{M-1} \\
& =\sum_{i=1}^{M} \frac{1}{i} \cdot\left(\begin{array}{c}
M-1 \\
i-1
\end{array}\right) \cdot \pi_{0}^{M-i} \cdot\left(1-\pi_{0}\right)^{i-1}
\end{aligned}
$$

The probability of receiving a packet from a parent is 0 if the parent buffer is empty. If it is not empty (i.e., in state $1-\pi_{0}$ ), the parent contends fairly with the other nodes that also have packet(s) buffered for transmission.

$$
\begin{aligned}
p_{1,0} & =0 \cdot \pi_{0}+\left(1-\pi_{0}\right)\left[1 \cdot \pi_{0}^{M-2}+\frac{1}{2} \cdot\left(\begin{array}{c}
M-2 \\
1
\end{array}\right) \cdot \pi_{0}^{M-3}\left(1-\pi_{0}\right)\right. \\
& +\frac{1}{3} \cdot\left(\begin{array}{c}
M-2 \\
2
\end{array}\right) \cdot \pi_{0}^{M-2}\left(1-\pi_{0}\right)^{2}+\ldots+ \\
& \left.+\frac{1}{M-1} \cdot\left(\begin{array}{c}
M-2 \\
M-2
\end{array}\right) \cdot\left(1-\pi_{0}\right)^{M-2}\right] \\
& =\left(1-\pi_{0}\right) \sum_{i=1}^{M-1} \frac{1}{i} \cdot\left(\begin{array}{c}
M-2 \\
i-1
\end{array}\right) \cdot \pi_{0}^{M-1-i} \cdot\left(1-\pi_{0}\right)^{i-1}
\end{aligned}
$$


If the child queue is not empty, it always contends for transmission with its parent. Thus $p_{1,+}$ is computed as follows:

$$
\begin{aligned}
p_{1,+} & =0 \cdot \pi_{0}+\left(1-\pi_{0}\right)\left[\frac{1}{2} \cdot \pi_{0}^{M-2}+\frac{1}{3} \cdot\left(\begin{array}{c}
M-2 \\
1
\end{array}\right) \cdot \pi_{0}^{M-3}\left(1-\pi_{0}\right)\right. \\
& +\frac{1}{4} \cdot\left(\begin{array}{c}
M-2 \\
2
\end{array}\right) \cdot \pi_{0}^{M-4}\left(1-\pi_{0}\right)^{2}+\ldots+ \\
& \left.+\frac{1}{M} \cdot\left(\begin{array}{c}
M-2 \\
M-2
\end{array}\right) \cdot\left(1-\pi_{0}\right)^{M-2}\right] \\
& =\left(1-\pi_{0}\right) \sum_{i=1}^{M-1} \frac{1}{i+1} \cdot\left(\begin{array}{c}
M-2 \\
i-1
\end{array}\right) \cdot \pi_{0}^{M-1-i}\left(1-\pi_{0}\right)^{i-1}
\end{aligned}
$$

The buffer at a node overflows when a node in state $\pi_{b_{i}}$ receives a packet. Thus,

$$
p_{o f}=\pi_{b_{i}} \cdot p_{1,+}
$$

The cost associated with this drop due to network resources already consumed by this packet increase with the hop distance of $n_{i}$ from the source along the routing path, i.e., packet dropped closer to source wastes fewer network resources compared to a packet dropped closer to destination. Assume the nodes $n_{i}$ are ordered such that they represent an increasing hop distance from the source, i.e., the hop count from the source for $n_{i}<n_{i+1}$. We can thus use the index $i$ for $i=\{1,2, \ldots, M\}$ as a simple cost function to represent the bias associated with a packet drop at node $n_{i}$.

Thus our optimization problem is as follows:

$$
\begin{array}{rc}
\min _{b_{i}} & \sum_{i=1}^{M} \pi_{b_{i}} \cdot p_{1,+} \cdot i \\
\text { subject to } & \sum_{i=1}^{M} b_{i}=B \\
\text { and } & b_{i} \geq 0, \forall i \in M
\end{array}
$$


The steady state flow balance leads to the following:

$$
\begin{aligned}
\pi_{0} \cdot p_{1,0} & =\pi_{1} \cdot p_{0} \\
\pi_{1} \cdot p_{1,+} & =\pi_{2} \cdot p_{0} \\
\cdots & \\
\pi_{b_{i-1}} \cdot p_{1,+} & =\pi_{b_{i}} \cdot p_{0}
\end{aligned}
$$

Therefore, we get

$$
\pi_{k}=\pi_{0}\left(\frac{p_{1,+}}{p_{0}}\right)^{k-1} \cdot \frac{p_{1,0}}{p_{0}}
$$

when $k>0$. Substituting this in $\sum_{k=0}^{b_{i}} \pi_{k}=1$, we can calculate $\pi_{0}$ since $p_{1,0}, p_{1,+}$, and $p_{0}$ can also be represented by $\pi_{0}$.

The above analysis is based on the assumption that all nodes experience identical transition probabilities. This assumption holds, irrespective of the buffer $b_{i}$ allocated to node $n_{i}$, as long as the probability of a node buffer being empty is small, i.e., $\pi_{0} \approx 0$. We can see that this allows $p_{0}, p_{1,0}$, and $p_{1,+}$ from Eqs. (4), (5), and (6) to converge to $\frac{1}{M}$ (for simplicity, we approximate $p_{1,0}$ from $\frac{1}{M-1}$ as $\frac{1}{M}$ ). This behavior is consistent with the 802.11 MAC that provides equal transmission opportunities (TXOPs) to all nodes in a collision domain.

From Eq. (9) we observe that $\pi_{b_{i}}=\frac{1}{b_{i}+1}$. Substituting into Eq. (7),

$$
p_{o f}=\pi_{b_{i}} \cdot p_{1,+}=\frac{1}{b_{i}+1} \cdot \frac{1}{M}
$$

Our optimization problem then becomes

$$
\begin{array}{rc}
\min _{b_{i}} & \sum_{i=1}^{M} \frac{1}{b_{i}+1} \cdot \frac{1}{M} \cdot i \\
\text { subject to } & \sum_{i=1}^{M} b_{i}=B \\
\text { and } & b_{i} \geq 0, \forall i \in \mathbf{M}
\end{array}
$$


The objective function in (11) can be expanded as follows:

$$
\frac{1}{M} \min _{b_{1}, b_{2}, \ldots, b_{M}}\left(\frac{1}{b_{1}+1}+\frac{2}{b_{2}+1}+\ldots+\frac{M}{b_{M}+1}\right)
$$

Let

$$
f \triangleq\left(\frac{1}{b_{1}+1}+\frac{2}{b_{2}+1}+\ldots+\frac{M}{b_{M}+1}\right)
$$

From analytical algebra, the optimal $b_{i}$ satisfies

$$
\frac{\partial f}{\partial b_{i}}=0, \forall i \in \mathbf{M}
$$

or the optimal $b_{i}$ are the boundary values, i.e., $b_{i}=0$ or $B$. Using substitution, we can verify that the boundary values do not minimize the cost function $f$. We

then turn to Eq. (14). This is a set of $M$ linear equations with $M$ unknowns whose solution is as follows:

$$
\begin{aligned}
b_{1} & =\frac{B+5}{1+\sqrt{2}+\sqrt{3}+\ldots+\sqrt{M}}-1 \\
b_{2} & =\frac{\sqrt{2}(B+5)}{1+\sqrt{2}+\sqrt{3}+\ldots+\sqrt{M}}-1 \\
\ldots & =\frac{\sqrt{M}(B+5)}{1+\sqrt{2}+\sqrt{3}+\ldots+\sqrt{M}}-1
\end{aligned}
$$

When $B$ is large, i.e., $B>>1$, we can approximate this as:

$$
b_{1}: b_{2}: \cdots: b_{M}=1: \sqrt{2}: \ldots: \sqrt{M}
$$

We note that the results in Eq. (16) hold for any cost function that increases linearly with the hop count.

\section{Implementation issues}

In this section we provide an overview of various implementation issues that need to be considered to provide a practical buffer sizing mechanism. 


\subsection{Determining network parameters}

A mesh node needs current network information to compute its buffer per our proposed framework. We provide guidelines to determine this information in an efficient, distributed manner.

Efficient techniques for building interference maps $[28,31]$ can be used to determine collision domains. Simpler models, like the two-hop interference model considered in this work, can be captured using local information at a node, e.g., using routing information to determine one and two-hop neighbors both up and down the routing tree, or monitoring the relay of traffic by neighboring nodes to determine two-hop neighbors. The utilization of each collision domain can then be monitored locally (e.g., Atheros hardware maintains a 32-bit register counter for medium busy utilization), and the collision domain with a utilization higher than some threshold (e.g., say 90\%) constitutes the bottleneck for a flow.

Once the collision domain is identified, wireless link rates can be inferred as follows: Device drivers maintain various wireless link statistics (including PHY rate information) between a node and its neighbors. Two-hop link rate information can be determined through capture and analysis of PHY frame header when the neighboring nodes relay the packet up or down the routing tree. This link rate information along with the packet size for a flow (again, known locally) is used to compute the RTT for propagating the packet through the collision domain.

The available BDP of a wireless network changes over time. Nodes recompute their buffer when network conditions change (e.g., changes in routing path or link rates) or when there are changes in flow activity information. This change in flow activity can be detected by a node using packet analysis for flows that originate locally or monitoring the wireless medium for flows in the neighborhood. If a flow is considered as a sum of various subflows originating from client devices associated with a mesh node, then this flow activity information may be stable for durations of tens of seconds (the large delay values discussed in this work are observed for long-lived flows such as large file transfers). A mesh node monitors its state for changes in network state or flow information, 


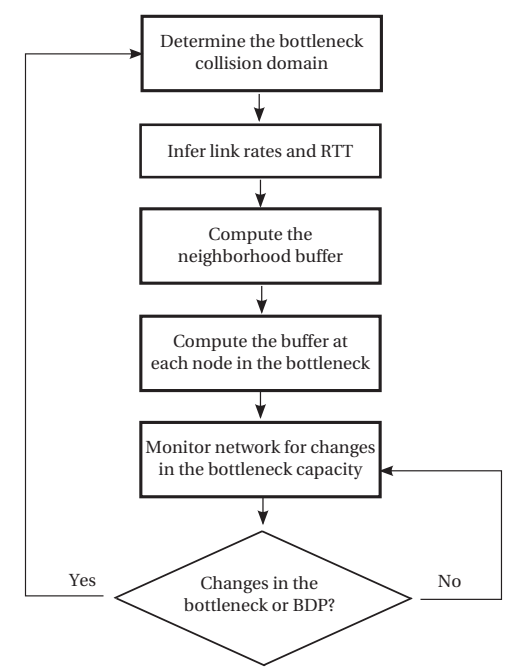

Figure 6: Flowchart for a heuristic buffer sizing technique.

and recomputes the buffer when necessary. A system flowchart for this cycle is shown in Fig. 6.

Finally, we note that inaccurate estimation of various network parameters yields suboptimal BDP for the bottleneck collision domain which is used to estimate the neighborhood and the per-node buffer. This suboptimal buffer size impacts the bandwidth-delay tradeoff performance for a flow. The approximations considered in Sect. 4 err on the side of overestimating BDP, thus preferring bandwidth at the cost of slight increase in end-to-end delay.

\subsection{Buffers in ns-2 and Linux network stack}

We evaluate our proposed buffer sizing strategy using simulations in ns-2 as well as experiments on a testbed deployment of 802.11 mesh routers running Linux kernel. In this section, we briefly describe the packet transmission buffers that we modify in our experiments.

\subsubsection{Buffers in $n s-2$}

A simplified architecture of the ns-2 wireless node is shown in Fig. 7a. The interface queue (IFQ) sits between the logical link layer and the MAC layer. The default queue object is a FIFO queue of size 50 packets. The MAC layer uses 


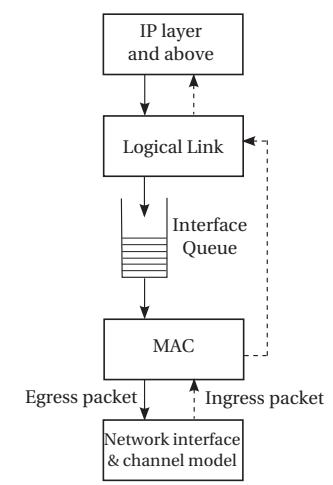

(a) ns-2 mobile node

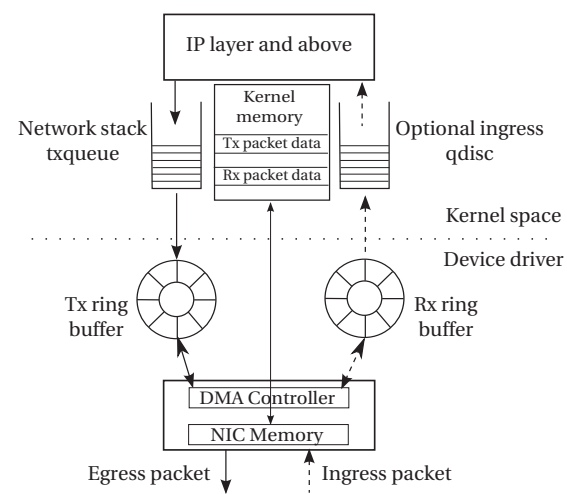

(b) Linux packet transmission and reception

Figure 7: Simplified architecture of buffers used in packet transmission/reception in a ns-2 mobile node and a Linux host.

callback functions to pull packets out of the IFQ when it is ready to transmit. We have instrumented a software framework to monitor the queue size of the IFQ and to modify it at run time per our framework requirements.

\subsubsection{Buffers in Linux network stack}

A packet transmission through a Linux host encounters buffers at multiple layers. At the radio interface side, there are hardware queues inside the physical adapter. Without these queues, the communication between the host and the adapter necessarily follows a 'stop-and-wait' approach, which is inefficient considering the large transfer latency between the host and the adapter. At the software side, the Linux network stack includes multiple layers of buffers. We describe the impact of some of these buffers on network performance.

Network stack's transmit queue: The Linux kernel introduces a transmit queue (txqueue) between the kernel network subsystem and the device drivers. This queue can then be scheduled by a rich set of queueing disciplines (qdiscs), providing a flexible traffic control framework.

Typical size: Most current Linux distributions set the default transmit queue length to 1000 packets. Older distributions generally used a 100 packet transmit queue for Fast Ethernet; the queue size has since been scaled up for 1 GigE interfaces. 
Impact of size: A large enough transmission queue is necessary to support line rate transfers for networks with high BDP. The value should be lowered for slower devices with a high latency as to prevent fast bulk transfers from disturbing real-time interactive traffic.

Device driver ring buffers Device drivers use queues to amortize interrupt processing overhead. This includes the Tx and Rx ring buffers (also called $\mathrm{Tx} / \mathrm{Rx}$ descriptors). Each descriptor represents a data structure that describes a buffer and its attributes to the network controller. The controller then uses this information to transfer data between the controller and the host memory. Typically device drivers have used a single Tx ring buffer. However, drivers with multiple queues are increasingly being used, e.g., 802.11 Enhanced Distributed Channel Access (EDCA) implementation uses separate queues for each of the four traffic access categories (ACs) in the standard: Background, Best effort, Video, and Voice. Typically, these traffic classes have different buffering requirements: real-time voice and video traffic prefers timeliness over reliability, and hence small buffers; background and best effort traffic can build relatively longer queues to take advantage of lull in transmission of higher priority traffic.

These queues may also be involved in performing MAC-specific tasks. For example, packet aggregation in $802.11 \mathrm{n}$ is performed at the MAC layer and thus needs a separate aggregation buffer. This buffer size is usually limited to a maximum of 64 MPDUs (MAC Protocol Data Units) as per the limitation of the block acknowledgment response frame as defined in the 802.11 standard specifications, though some drivers use smaller values.

Typical buffer sizes: The Tx/Rx ring size is driver dependent. In Table 2 we show the default ring buffer size for ath5k and ath9k drivers in the 2.6.35 kernel. The mac80211 Linux subsystem supports drivers with up to 4 queues per EDCA ACs. ath5k drivers have a total buffer size of 200 packets divided equally among the 4 ACs. ath9k drivers similarly equally share a buffer size of 512 packets among the ACs.

Impact of size: These values reflect various design and performance considerations. On one hand, memory consumption can be lowered by using small 


\begin{tabular}{l|c|c}
\hline Driver & Tx descriptors & Rx descriptors \\
\hline \hline ath5k (Atheros $802.11 \mathrm{a} / \mathrm{b} / \mathrm{g})$ & $200(50$ for each $\mathrm{AC})$ & 40 \\
\hline ath9k $($ Atheros $802.11 \mathrm{n})$ & $512(128$ for each $\mathrm{AC})$ & 512 \\
\hline
\end{tabular}

Table 2: DMA ring buffers in stock 2.6.35 Linux kernel

buffers. However, if the system is CPU bound or has a saturated memory bus, then small buffers may result in dropped or missed packets. The mesh routers used in our testbed deployment are not CPU or memory bound (our system hardware specifications are listed in Sect. 7), and thus buffer sizing considerations ignore hardware bottlenecks.

\subsubsection{Which buffer to tune?}

In this work, we keep the device driver Tx ring buffers at a constant value and tune the txqueue size per our framework requirements. This decision was made, in part, to maintain some buffers at the transmit queue layer so as to allow the flexibility of using Linux's traffic control framework, if required. We vary the size of the txqueue buffer using the if config userspace utility. We fix the size of our Tx ring descriptors to a static value of 3 packets by extending the Linux ethtool utility. We found that setting the Tx ring descriptors to a lower value interferes with timely transmission of 802.11 s management frames during the course of our experiments, leading to link disassociation and subsequent network fragmentation. Thus, in our experiments, we tune a mesh node's buffer size to be 3 packets larger than that predicted by the model in Sect. 4 .

\section{Performance Analysis - Simulations}

We benchmark our performance results against the following: (1) A 50packets buffer at all mesh routers. This is the default ns-2 configuration commonly used in research publications; (2) TCP-AP [18], a TCP pacing mechanism for multihop wireless networks (see Sect. 2 for a brief description of TCP-AP). Pacing TCP traffic minimizes bursts that lead to large queueing delays. TCPAP paces its traffic using 4-hop propagation delay, thus minimizing queueing 


\begin{tabular}{l|c|c}
\hline Scheme & Goodput Kb/s & Mean RTT (Std. Dev.) ms \\
\hline \hline 50 pkts buffer & 930 & $316(68)$ \\
\hline TCP-AP & 850 & $15(0.5)$ \\
\hline Buffer per Eq. (3) \& (16) & 878 & $22(2)$ \\
\hline
\end{tabular}

Table 3: Performance comparison for a 4-hop TCP flow with $11 \mathrm{Mb} / \mathrm{s}$ wireless links. All nodes are within mutual carrier sense range.

along the interfering links. Consistent with [18], we use a 50-packets buffer for TCP-AP, though we find that queues hardly build up beyond a few packets. We simulate backlogged TCP traffic with a large file transfer. The TCP segment size used in our simulations is 1460 bytes. Wireless link rates are $11 \mathrm{Mb} / \mathrm{s}$, unless specified otherwise. RTS/CTS control frames are disabled.

\subsection{Single flow topologies}

We first analyze two variants of a 4-hop chain: (1) when all nodes are within mutual carrier sense range, and (2) when the edge nodes are outside carrier sense range. The two scenarios experience different sources of packet loss: queue overflows in the first topology (as most collisions are resolved by MAC-layer ARQs), and collisions due to asymmetric information about the channel state [20] in the latter. The neighborhood buffer size $B$ computed per Eq. (3) depends on the wireless link rates, e.g., $B \approx 15$ packets for $54 \mathrm{Mb} / \mathrm{s}$ links $(802.11 \mathrm{a} / \mathrm{g})$ or $B \approx 85$ packets for $600 \mathrm{Mb} / \mathrm{s}$ links (802.11n). For $11 \mathrm{Mb} / \mathrm{s}$ links (802.11b) used in our simulations, $B \approx 10$ packets. Per Eq. (16), we distribute it as buffer of size 2, 2, 3, and 3 packets at 1, 2, 3, and 4-hop nodes from the source, respectively. For symmetric wireless links, the performance for upload flows is similar to that obtained for download flows between the gateway and a given mesh node. The gateway bridges this traffic to a server on the wired network via its $1 \mathrm{~Gb} / \mathrm{s}$ Ethernet interface.

Our results are summarized in Tables 3 and 4 . They confirm that the base case with a 50-packets buffer yields the highest aggregate throughput, albeit with large RTT delays. This may be immaterial for large file transfers where the objective is to maximize throughput. Regardless, a network should be able 


\begin{tabular}{l|c|c}
\hline Scheme & Goodput Kb/s & Mean RTT (Std. Dev.) ms \\
\hline \hline 50 pkts buffer & 910 & $200(49)$ \\
\hline TCP-AP & 825 & $15(0.5)$ \\
\hline Buffer per Eq. (3) \& (16) & 830 & $23(1.3)$ \\
\hline
\end{tabular}

Table 4: Performance comparison for a 4-hop TCP flow with $11 \mathrm{Mb} / \mathrm{s}$ wireless links. The two end nodes outside mutual carrier sense range.

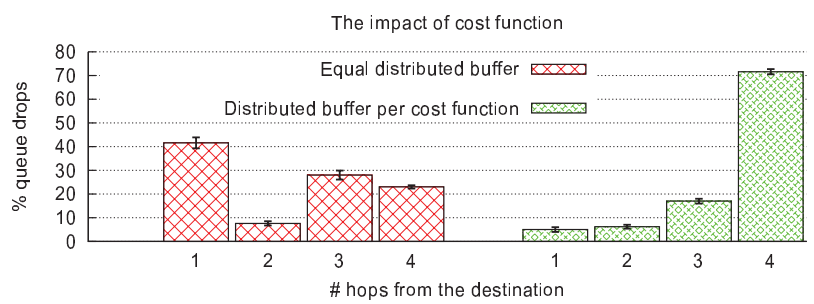

Figure 8: Queue drops at mesh routers in a 4-hop chain

to provide low-delay services, especially when TCP flows share buffers with realtime flows or short-lived TCP flows. Thus, by preventing long-lived flows from saturating large buffers, we indirectly improve the QoS characteristics of both real-time and short-lived flows. Our proposed scheme lowers the base case RTT by a factor of 15 and 9 across the two topologies, at the cost of about $6 \%$ to 9\% drop in throughput. TCP-AP achieves the lowest delay among the three schemes. In general, however, there are practical limitations of deploying pacing in real networks: First, while WMN service providers can configure the buffers on their mesh routers, but they often have no control on the TCP/IP stack on the client devices. Second, paced flows fare poorly when sharing a network with non-paced flows [10], thus limiting the incentive for a user to migrate. We evaluate this further for multi-flow topologies in Sect. 6.2 below.

We further analyze the performance improvements of our proposed cost function. Using a 4-hop chain topology, we first distribute a 12-packet neighborhood buffer equally among the relay nodes (a 3-packet buffer at each relay) and compare its performance to a buffer distribution using our proposed cost function. The experiment was repeated 5 times; we show the average queue drops (with the standard deviation) at each node in Fig. 8. With equal-sized buffers, up 


\begin{tabular}{l|c|c}
\hline Scheme & Normalized Goodput & Normalized RTT \\
\hline \hline 50 pkts buffer & 1 & 20.3 \\
\hline TCP-AP & 0.90 & 1 \\
\hline Buffer per Eq. (3) \& (16) & 0.96 & 2.2 \\
\hline
\end{tabular}

Table 5: Performance statistics averaged over multiple topologies with varying hop count. Goodput results are normalized to the goodput achieved with the base case for a 50 packet buffer for that simulation. RTT results are normalized to the RTT measured with TCP pacing for that simulation. We present averages computed over multiple different topologies.

to $42 \%$ queue drops happen at the node 1-hop away from the destination. In contrast, with our proposed cost function, over $70 \%$ of the queue drops occur at the node closest to the source. In this set of simulations, our scheme further reduced RTT by about $10 \%$ with a slight improvement in throughput of $2-3 \%$.

We have validated our proposed buffer sizing approach on multiple other topologies by varying the flow hop count, including topologies where packet losses occur due to both buffer overflows as well as collisions. In all simulations, the base case with the 50-packets buffer yielded the highest goodput, while TCP-AP yielded the least delay. We normalize the goodput and RTT for a given simulation run to these values, and then average those up over different topologies. Our results are tabulated in Table 5. In general, our proposed buffer sizing strategy achieves about $96 \%$ goodput of the base case with a 50-packets buffer, while reducing the RTT by a factor of 10 .

\subsection{Multi-flow topologies}

Our problem formulation in Sect. 4 considers a single TCP stream. We now investigate if the underlying behavior is also sustainable in a multi-flow network. Our motive here is not to determine the optimal cumulative neighborhood buffer in multi-flow environments (we defer this to future work); instead, we simply wish to evaluate whether small buffers also work well with multiple flows. We consider two illustrative scenarios: (1) a parking lot topology where multiple flows pass through shared relay nodes, and (2) a cross topology when multiple flows share the bottleneck spectrum but not the relay nodes. We perform ex- 


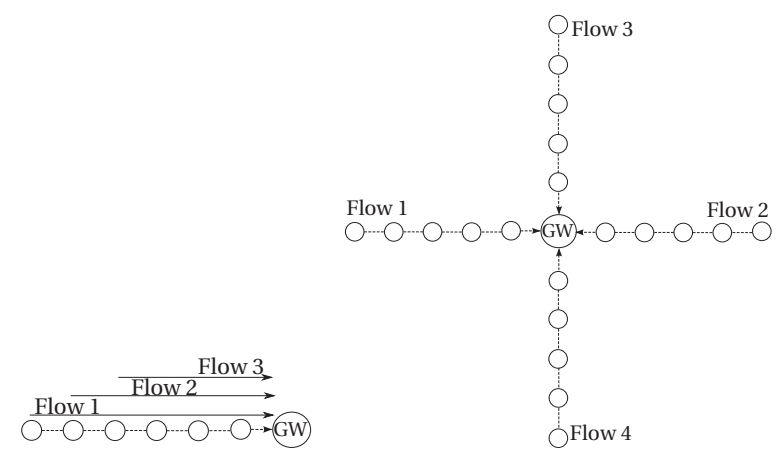

(a) Parking lot topology

(b) Cross topology

Figure 9: Multi-flow topologies

periments in which each flow represents either (i) a large file transfer with TCP, or (ii) a bundle containing a large file transfer with TCP and a VoIP stream. We simulate VoIP streams with G.729 codec characteristics using constant bit rate (CBR) UDP traffic: a stream bit rate of $8 \mathrm{~Kb} / \mathrm{s}$ with a packet size of 40 bytes each. For toll quality service, VoIP streams should maintain a one-way latency (mouth to ear) of less than $150 \mathrm{~ms}$ and a delay jitter of less than 30 ms [32].

Parking lot topology We first experiment with the parking lot topology in Fig. 9a. It has data flows originating from nodes 4, 5, and 6-hop away from the gateway. These flows share a common bottleneck collision domain. Considering each flow in isolation, our buffer sizing strategy in Sect. 4 can be used to compute a neighborhood buffer size $B_{1}, B_{2}$, and $B_{3}$ corresponding to each flow. We then distribute these buffers such that a mesh node in the collision domain has a per-flow buffer of size determined by Eq. (16). Per-flow queueing requires flow classification at relay nodes; we perform it using source node IP address and port numbers. We service these queues using fair queueing [16] on top of DCF scheduling. Our methodology considerably overestimates the cumulative buffer required for saturating the bottleneck spectrum; however, the per-flow buffer (of 1-3 packets) at each relay node is still small. Determining the optimal buffer size in such multi-flow networks is left for future work. 


\begin{tabular}{l|c|c}
\hline Scheme & Avg. Goodput (Std. Dev.) Kb/s & Mean RTT (Std. Dev.) ms. \\
\hline \hline 50 pkts buffer & $285(68)$ & $354(35)$ \\
\hline TCP-AP & $264(32)$ & $38(3)$ \\
\hline Buffer per Eq. (3) \& (16) & $275(22)$ & $58(3)$ \\
\hline
\end{tabular}

Table 6: Parking lot topology. 3 FTP streams

\begin{tabular}{l|l|l|l|l|l}
\hline \multirow{2}{*}{ Scheme } & \multicolumn{2}{|c|}{ FTP } & \multicolumn{2}{c}{ VoIP } \\
\cline { 2 - 6 } & $\begin{array}{l}\text { Mean Goodput } \\
\text { (Std. Dean }\end{array}$ & $\begin{array}{l}\text { MTT } \\
\text { (Std. Dev.) } \\
\text { ms. }\end{array}$ & $\begin{array}{l}\text { Mean Bit Rate } \\
\text { Kb/s }\end{array}$ & $\begin{array}{l}\text { Mean Delay } \\
\text { (Jitter) ms. }\end{array}$ \\
\hline \hline 50 pkts buffer & $261(58)$ & $388(32)$ & 7.8 & $239(8)$ \\
\hline TCP-AP & $240(17)$ & $54(6)$ & 8 & $37(5)$ \\
\hline Buffer per Eq. (3) \& (16) & $250(33)$ & $87(6)$ & 8 & $40(6)$ \\
\hline
\end{tabular}

Table 7: Parking lot topology. 3 FTP and 3 VoIP streams

Our results for the three large file transfer streams are summarized in Table 6. We list the mean and the standard deviation for goodput and RTT averaged over the three flows. TCP pacing lowers RTT by a factor of 9 at a cost of $8 \%$ drop in goodput. In contrast, our distributed buffering strategy improves RTT by a factor of 6 at the cost of less than $4 \%$ drop in average goodput. These improvements may appear relatively modest compared to our results for networks with single TCP streams. This is partly because multiple streams sharing a 50 packet buffer do not let TCP congestion window for individual streams grow quickly to unsustainable large values. Finally, our improvements with distributed buffers are obtained despite the suboptimal (though small) buffer sizes used in this experiment.

As mentioned earlier, paced TCP traffic fares poorly when competing with non-paced TCP streams. We highlight this using a TCP-AP stream for the 4-hop flow and non-paced TCP for the 5 and 6-hop flows in Fig. 9a. Our results show that the paced flow achieves less than $\frac{1}{10}$ th of the throughput of the other flows. No such unfairness is observed when none of the three flows are paced. Note that this behavior is very different from the unfairness observed in prior work [20], as it is the stream closest to the gateway that is losing throughput. This performance challenge is an impediment in wide-spread deployment of pacing-based protocols. 
Table 7 shows our results when each flow in Fig. 9a represents a bundle containing a large file transfer with TCP and a VoIP stream. For the VoIP traffic, we list the mean bit rate (stream goodput), one way delay, and delay jitter averaged over the three streams. With large network buffers, TCP streams can quickly build a large congestion window. This increases the queueing delay for the VoIP traffic that shares the buffer with the TCP streams. With 50 packet buffers, the average delay for the three VoIP streams far exceeds the 150 ms delay bound. In contrast, both TCP-AP and our proposed distributed buffer sizing mechanism limit this unchecked growth of the TCP congestion window. TCP-AP throttles the TCP streams more aggressively, roughly incurring a loss of $5 \%$ in goodput compared to our proposed buffer sizing mechanism. As a final observation, we note that the combined goodput of the FTP and VoIP streams in Table 7 is less than the corresponding entries in Table 6 . This is because the 802.11 overhead is better amortized over the larger 1460 byte TCP segments used in our FTP simulations. Various optimizations for reducing this overhead have been discussed in prior work [21].

\begin{tabular}{l|c|c}
\hline Scheme & Mean Goodput (Std. Dev.) Kb/s & Mean RTT (Std. Dev.) ms. \\
\hline \hline 50 pkts buffer & $465(44)$ & $260(53)$ \\
\hline TCP-AP & $417(24)$ & $28(5)$ \\
\hline Buffer per Eq. (3) \& (16) & $455(27)$ & $56(13)$ \\
\hline
\end{tabular}

Table 8: Cross topology. 4 FTP streams

\begin{tabular}{l|l|l|l|l}
\hline \multirow{2}{*}{ Scheme } & \multicolumn{2}{|c|}{ FTP } & \multicolumn{2}{c}{ VoIP } \\
\cline { 2 - 6 } & $\begin{array}{l}\text { Mean Goodput } \\
\text { (Std. Dev.) } \\
\text { Kb/s }\end{array}$ & $\begin{array}{l}\text { Mean RTT } \\
\text { (Std. Dev.) } \\
\text { ms. }\end{array}$ & $\begin{array}{l}\text { Mean Bit Rate } \\
\text { Kb/s }\end{array}$ & $\begin{array}{l}\text { Mean Delay } \\
\text { (Jitter) ms. }\end{array}$ \\
\hline \hline 50 pkts buffer & $382(17)$ & $309(72)$ & 7.8 & $187(30)$ \\
\hline TCP-AP & $339(15)$ & $33(6)$ & 7.9 & $24(4)$ \\
\hline Buffer per Eq. (3) \& (16) & $368(5)$ & $71(8)$ & 7.9 & $35(4)$ \\
\hline
\end{tabular}

Table 9: Cross topology. 4 FTP and 4 VoIP streams

Cross topology We next consider the cross topology in Fig. 9b. A source node 5-hops along each edge sends a data flow to the gateway in the center. Using a 


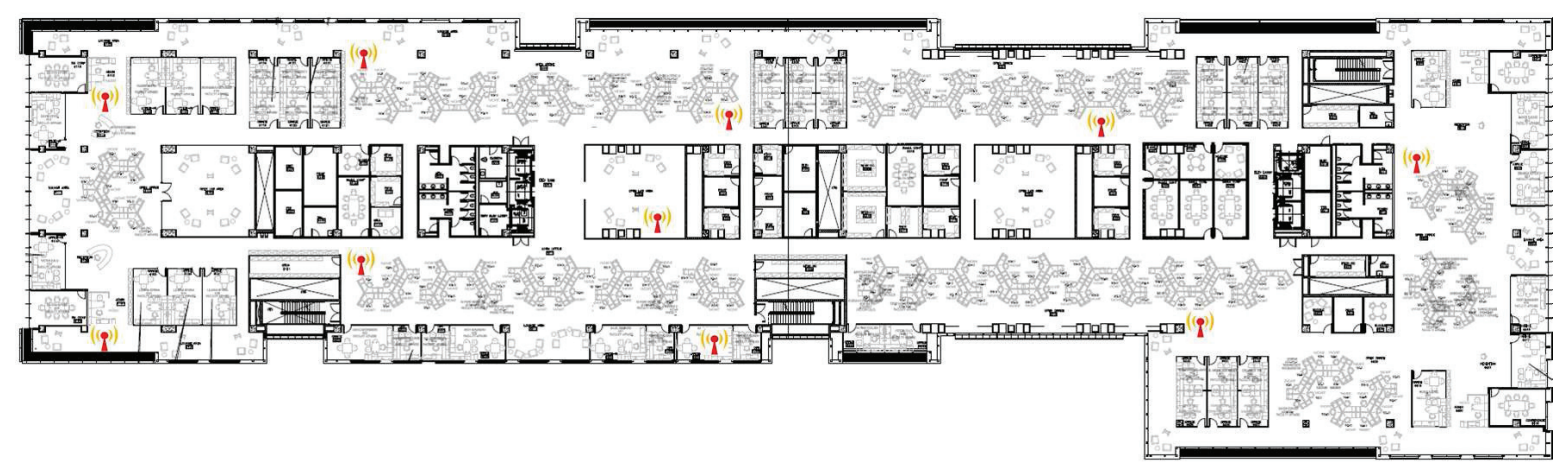

Figure 10: Mesh node positions (identified by a radio icon) in our WMN testbed 2-hop interference model, the 1 and 2-hop nodes around the gateway along each edge constitute the shared bottleneck collision domain between the four flows. With uniform $11 \mathrm{Mb} / \mathrm{s}$ links, this distributed buffer size is $B=18$ packets per Eq. 3. Since the number of hops (and consequently the cost of packet drop) are similar for each edge, we distribute $B$ as 2 and 3 packets at the 3 and 4-hop nodes from source along each edge.

Our results for the FTP streams are summarized in Table 8. We list the mean and the standard deviation for the four flows. TCP pacing lowers RTT 10 -fold at a cost of $10 \%$ drop in goodput. Our distributed buffering strategy improves RTT by $5 \times$ with $2 \%$ drop in average goodput of the four flows.

Table 9 shows our results when each flow in Fig. $9 \mathrm{~b}$ represents a bundle containing a large file transfer with TCP and a VoIP stream. Consistent with our observations for the parking lot topology, TCP fills up available network buffers, leading to unacceptable VoIP delays when large network buffers are shared with the TCP stream. Right sizing the network buffers per our proposed mechanism reduces the VoIP delays by more than a factor of 5 , at a cost of less than $3 \%$ loss in goodput for the TCP streams.

\section{Performance Analysis - Testbed}

We have validated the performance of our proposed buffer sizing mechanism on a 10-node WMN testbed in our university campus. Node locations are 
shown in Fig. 10 and were determined, in part, by availability of power and Ethernet drops. Each mesh router is a small form-factor Shuttle [8] computer with an Intel E7500 Core 2 Duo processor, 1 GB of DRAM, and a TP-Link WN350G (Atheros AR2417) 802.11 b/g card. Our network uses the $2.4 \mathrm{GHz}$ ISM (Industrial, Scientific, and Medical) band and shares the spectrum with the production wireless LAN in our campus. We run a custom 2.6.35 Linux kernel with web100 [9] instrumentation to monitor the state characteristics of our TCP streams. We use ath5k drivers [1] to configure our wireless interface. Our platform runs the open80211s [7] implementation of the 802.11s standard. This was partly driven by our desire to understand and contribute to the ongoing development of the framework. Our testbed uses HWMP (Hybrid Wireless Mesh Protocol) routing protocol with airtime metric [14] for path selection. We use static wireless link rates for a given experiment. This ensures that any performance improvements come from buffer sizes and not from dynamic changes in link rate. RTS/CTS control frames are disabled.

We use iperf [4] to generate test traffic. We generate TCP streams with a segment size of 1448 bytes. The kernel then couples this with a 20-byte TCP header, a 10-byte TCP timestamp option header, a 2-byte padding field, and a 20-byte IPv4 header for a total maximum segment size (MSS) of 1500 bytes. Cubic is the default TCP congestion control algorithm in Linux kernels since 2.6.18 [22]. It replaces the linear window growth function of TCP variants such as NewReno and SACK by a cubic function so as to better improve the utilization of long fat networks (LFNs). In future work, we intend to evaluate our proposed scheme with other TCP variants used in commodity OS such as Windows.

We have performed multiple experiments to evaluate our proposed buffer sizing framework. For comparative analysis, we benchmark our performance results with the default buffer sizes used in our Linux distribution. In our first set of experiments, we varied the hop length of a path from 2 to 4 hops. These experiments were repeated along various source and destination pairs across the testbed to offset any location-specific wireless idiosyncrasies. For a 3-hop chain 
and $11 \mathrm{Mb} / \mathrm{s}$ wireless links, the neighborhood buffer size $B$ computed from Eq. (3) is approximately 8 packets. Per Eq. (16), we distribute it as a txqueue buffer of size 2, 3, and 3 packets at 1, 2, and 3-hop nodes from the source, respectively. The buffer sizes for other topologies were similarly calculated. Our results are shown in Fig. 11. We normalize the goodput measured with our proposed buffer sizing mechanism to the goodput for the same topology with default buffer sizes in our Linux distribution. For RTT, we normalize the delays with large buffers to the delay values measured with our proposed mechanism. The error bars in these graphs are the 95\% confidence intervals. Our results show a two-orders of magnitude improvement in two-hop and $25 \times$ improvement in three-hop delays while obtaining upwards of $92 \%$ goodput achieved with large buffers in both cases. Our results with a 4 -hop chain show a $6 \times$ improvement in delay while achieving $90 \%$ of the large-buffer goodput. These results show that wireless specific losses are minimal in smaller topologies (thanks to MAC-level retransmissions in 802.11) where most nodes can carrier sense each other, and hence TCP congestion avoidance algorithms mostly reacts to losses when large buffers overflow. In contrast, in larger topologies wireless losses tend to dominate and prevent TCP congestion window from completely filling up intermediate router buffers. However, the window still grows to suboptimal large values that increases queueing delays beyond those acceptable for real-time traffic, e.g., in our 4-hop topology, the average RTT with large buffers was $431 \mathrm{~ms}$ as compared to $67 \mathrm{~ms}$ with our proposed buffer sizing mechanism.

We now investigate if the underlying performance improvements are also sustainable in a multi-flow network using small buffers. Our next set of experiments considers a 3-hop and a 4-hop flow in our 10-node testbed. The two flows traverse disparate (though interfering) nodes along their path to the destination. Considering each flow in isolation, our buffer sizing strategy in Sect. 4 can be used to compute a neighborhood buffer size $B_{1}$ and $B_{2}$ corresponding to each flow. We then distribute these buffers such that a mesh node in the collision domain has a per-flow buffer of size determined by Eq. (16).

Our results are summarized in Table 10. We list the mean goodput and the 


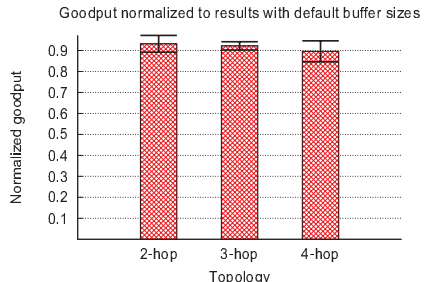

(a) Flow goodput

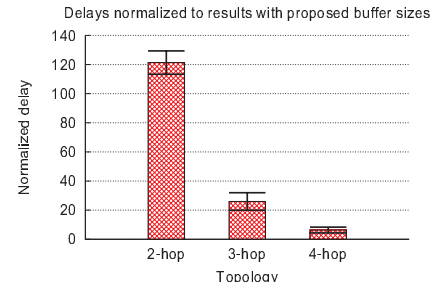

(b) RTT

Figure 11: Flow goodput and delay across various testbed topologies. We normalize the goodput with default buffer sizes to the goodput achieved with our proposed mechanism. For RTT, we normalize the RTT measurements with default buffers to the RTT measured with our proposed mechanism. Error bars are the $95 \%$ confidence intervals.

\begin{tabular}{l|c|c}
\hline Scheme & Avg. Goodput (Std. Dev.) Kb/s & Mean RTT (Std. Dev.) ms. \\
\hline \hline Default, large buffers & $786(45)$ & $1653(327)$ \\
\hline Buffer per Eq. (3) \& (16) & $712(6)$ & $91(19)$ \\
\hline
\end{tabular}

Table 10: Multi-flow experimental results. A 3-hop and a 4-hop flow together share the network along disjoint paths.

RTT for the two flows, as well as their standard deviation. Our proposed buffer sizing mechanism reduces the average RTT of the two flows by a factor of 20 , at the cost of about $9 \%$ drop in aggregate goodput.

\begin{tabular}{l|c|c}
\hline Scheme & Avg. Goodput (Std. Dev.) Mb/s & Mean RTT (Std. Dev.) ms. \\
\hline \hline Default, large buffers & $8.8(0.3)$ & $1113(91)$ \\
\hline Buffer per Eq. (3) \& (16) & $8.1(0.04)$ & $153(9)$ \\
\hline
\end{tabular}

Table 11: A 4-hop 802.11n mesh topology connected via $65 \mathrm{Mb} / \mathrm{s}$ links.

Finally, we have also validated our proposed buffer sizing mechanism for 802.11n networks. We swapped the TP-Link WN350G wireless adapters with TP-Link TL-WDN4800 (AR 9380 chipset) radios on a subset of our testbed nodes. In this set of experiments, we used download TCP flows (file transfer from the server to the wireless client) over a 4-hop chain topology. Our results are summarized in Table 11. With $65 \mathrm{Mb} / \mathrm{s}$ links, the default buffer size in Linux yields an average throughput of $8.8 \mathrm{Mb} / \mathrm{s}$ with a mean RTT of $1113 \mathrm{~s}$ 
over the 4-hop topology. With our proposed scheme, we obtain a neighborhood buffer of approximately 14 packets. Using the linear cost function in Section 4.2 to distribute the buffers over the bottleneck, we observe a $7 \times$ reduction in RTT at the cost of about $8 \%$ drop in flow throughput.

\section{Conclusions and Open Issues}

Buffer sizing has extensively been studied in the context of core Internet routers handling a large number of flows. These networks have a large BDP, and even optimizations suggested in recent work lead to buffer sizes in MBs. Wireless networks, however, can have a much smaller BDP, translating to small buffer sizes. Configuring these buffer sizes in commodity OS (such as Linux) is a challenge because buffers are spread over multiple layers in the software stack. We describe the major sources of buffering encountered by a packet through a Linux kernel. Through careful sizing of various buffers, our goal is to achieve high utilization of the bottleneck spectrum while maintaining low queueing delays. In this context, we use the concept of a neighborhood buffer distributed over multiple nodes to determine the cumulative buffer size that will saturate the spectral resource constituting the network bottleneck. We propose

heuristics for sizing the cumulative neighborhood buffer, and for distributing it among the contending nodes. Our evaluation using simulations and testbed experiments show that we can effectively maintain high network utilization with low delays.

There are interesting avenues for future work in the area. We are currently investigating the impact of Aggregate MAC Protocol Data Unit (A-MPDU) frame scheduling on packet delays in 802.11n networks. Further, in this work we distribute the neighborhood buffer among the nodes using a simple cost function where the cost of a packet drop along a path increases linearly with the hop count. With multi-rate hops along a path, this cost may not increase linearly. Other alternatives, such as dropping packets only on the first hop, may perform better. We intend to explore this in future work. Finally, our current 
buffering strategy uses a loose upper bound on achievable network capacity to size the neighborhood buffer. We are working on an adaptive approach in which the nodes in a radio neighborhood measure the current flow rates to estimate the network carrying capacity and adjust their allocated buffer sizes in response. We anticipate that these adaptive schemes would fare better in multi-flow networks as well as in wireless networks susceptible to large variability in channel noise and interference.

\section{References}

[1] ath5k FOSS drivers. http://wireless.kernel.org/en/users/Drivers/ ath5k.

[2] ath9k FOSS drivers. http://wireless.kernel.org/en/users/Drivers/ ath9k.

[3] Bufferbloat. http://www.bufferbloat.net/.

[4] iperf. http://sourceforge.net/projects/iperf/.

[5] Madwifi: Multiband Atheros driver for WiFi. http://www. madwifi-project.org.

[6] The network simulator - ns-2. http://www.isi.edu/nsnam/ns.

[7] open80211s. http://open80211s.org.

[8] Shuttle Inc. http://www. shuttle.com.

[9] The web100 project. http://www.web100.org/.

[10] A. Aggarwal, S. Savage, and T. Anderson. Understanding the performance of TCP pacing. In Proc. of the IEEE INFOCOM '00, pages 1157-1165, Mar. 2000.

[11] G. Appenzeller, I. Keslassy, and N. McKeown. Sizing router buffers. In Proc. of the ACM SIGCOMM '04, pages 281-292, Sept. 2004. 
[12] H. Balakrishnan, C. L. Barrett, V. S. A. Kumar, M. Marathe, and S. Thite. The distance-2 matching problem and its relationship to the MAC-layer capacity of ad hoc networks. IEEE Journal on Selected Areas in Communication, 22(6):1069-1079, Aug. 2004.

[13] R. Bruno, M. Conti, and E. Gregori. Analytical modeling of TCP clients in Wi-Fi hot spot networks. In Proc. of the IFIP Networking '04, pages 626-637, May 2004.

[14] J. Camp and E. Knightly. The IEEE 802.11s extended service set mesh networking standard. IEEE Communications Magazine, 46:120-126, Aug. 2008.

[15] K. Chen, Y. Xue, S. Shah, and K. Nahrstedt. Understanding bandwidthdelay product in mobile ad hoc networks. Elsevier Computer Communications, 27(10):923-934, June 2004.

[16] A. J. Demers, S. Keshav, and S. Shenker. Analysis and simulation of a fair queueing algorithm. In Proc. of the ACM SIGCOMM '89, pages 1-12, Sept. 1989.

[17] O. Dousse. Revising buffering in CSMA/CA wireless multihop networks. In Proc. of the IEEE SECON 'O\%, June 2007.

[18] S. M. ElRakabawy, A. Klemm, and C. Lindemann. TCP with adaptive pacing for multihop wireless networks. In Proc. of the ACM MobiHoc '05, pages 288-299, May 2005.

[19] M. Enachescu, Y. Ganjali, A. Goel, N. McKeown, and T. Roughgarden. Routers with very small buffers. In Proc. of the IEEE INFOCOM '06, Apr. 2006.

[20] V. Gambiroza, B. Sadeghi, and E. Knightly. End-to-end performance and fairness in multihop wireless backhaul networks. In Proc. of the ACM MobiCom '04, pages 287-301, Sept. 2004. 
[21] S. Ganguly, V. Navda, K. Kim, A. Kashyap, D. Niculescu, R. Izmailov, S. Hong, and S. R. Das. Performance optimizations for deploying VoIP services in mesh networks. IEEE Journal on Selected Areas in Communications, 24(11):2147-2158, 2006.

[22] S. Ha, I. Rhee, and L. Xu. CUBIC: a new TCP-friendly high-speed TCP variant. SIGOPS Oper. Syst. Rev., 42:64-74, July 2008.

[23] B. Hull, K. Jamieson, and H. Balakrishnan. Mitigating congestion in wireless sensor networks. In Proc. of the ACM SenSys '04, Baltimore, MD, Nov. 2004.

[24] IEEE LAN/MAN Standards Committee. IEEE 802.11 - Wireless LAN medium access control (MAC) and physical layer (PHY) specifications. IEEE, June 2007.

[25] K. Jamshaid, B. Shihada, L. Xia, and P. Levis. Buffer sizing in 802.11 wireless mesh networks. In Proc. of the IEEE MASS '11, pages 272-281, Oct. 2011.

[26] R. Johari and D. Tan. End-to-end congestion control for the internet: delays and stability. IEEE/ACM Transactions on Networking, 9(6):818832, Dec. 2001.

[27] J. Jun and M. L. Sichitiu. The nominal capacity of wireless mesh networks. IEEE Wireless Communications, pages 8-14, Oct. 2003.

[28] A. Kashyap, U. Paul, and S. R. Das. Deconstructing interference relations in WiFi networks. In Proc. of the IEEE SECON '10, pages 73-81, June 2010.

[29] T. Li, D. J. Leith, and D. Malone. Buffer sizing for 802.11-based networks. IEEE/ACM Transactions on Networking, 19(1):156-169, Feb. 2011.

[30] J. Padhye, S. Agarwal, V. N. Padmanabhan, L. Qiu, A. Rao, and B. Zill. Estimation of link interference in static multi-hop wireless networks. In Proc. of the ACM/USENIX IMC' '05, pages 305-310, Oct. 2005. 
[31] L. Qiu, Y. Zhang, F. Wang, M. Han, and R. Mahajan. A general model of wireless interference. In Proc. of the ACM MobiCom '07, pages 171-182, Sept. 2007.

[32] T. Szigeti and C. Hattingh. End-to-end QoS network design: Quality of Service in LANs, WANs, and VPNs. Cisco Press, 1st edition, 2004.

[33] C. Villamizar and C. Song. High performance TCP in ANSNET. SIGCOMM Computer Communications Review, 24(5):45-60, 1994.

[34] K. Xu, M. Gerla, L. Qi, and Y. Shu. Enhancing TCP fairness in ad hoc wireless networks using neighborhood RED. In Proc. of the ACM MobiCom '03, pages 16-28, Sept. 2003.

[35] L. Zhang, S. Shenker, and D. D. Clark. Observations on the dynamics of a congestion control algorithm: the effects of two-way traffic. In Proc. of the ACM SIGCOMM '91, pages 133-147, Oct. 1991. 
Kamran Jamshaid received a Ph.D. in Electrical and Computer Engineering from University of Waterloo, MS in Computer Science from Wayne State University, and a BS in Electronic Engineering from GIK Institute, Pakistan. His research interests include computer networks, distributed systems, and pervasive and mobile computing. 
${ }^{*}$ Author Photo

Click here to download high resolution image

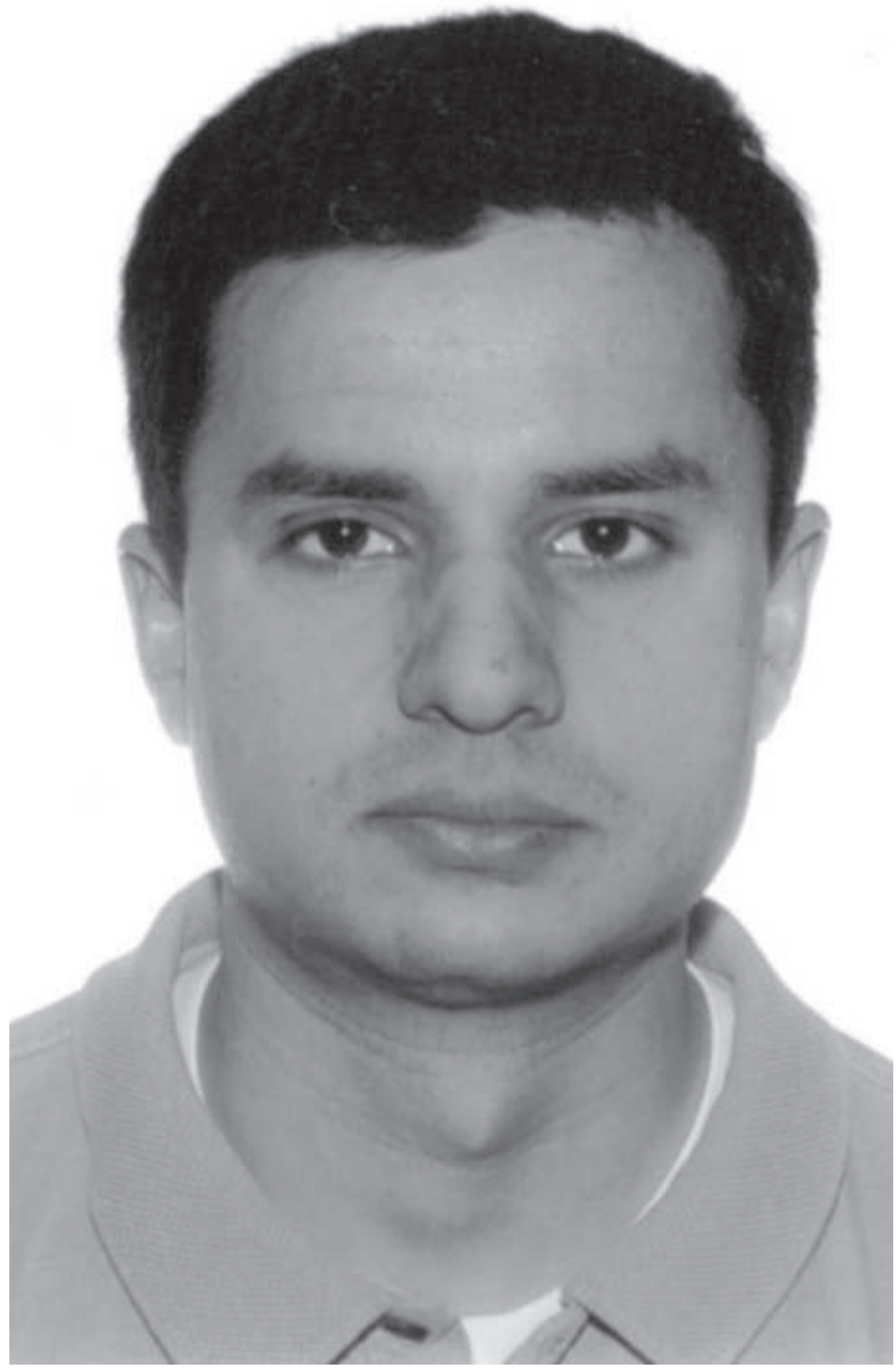

